\title{
Oridonin Attenuates Low Shear Stress-Induced Endothelial Cell Dysfunction and Oxidative Stress by Activating The Nuclear Factor Erythroid 2-Related Factor 2 Pathway
}

\section{ZHIPENG CHEN}

Affiliated Drum Tower Hospital, Medical School of Nanjing University

HEQIAN LIU

Xuzhou Medical College

SUBINUR MAMATELI

Affiliated Drum Tower Hospital, Medical School of Nanjing University

CHENG LIU

Nanjing Drum Tower Hospital

YUTONG LIU

Nanjing University

JING CAI

Nanjing Drum Tower Hospital

TONG QIAO ( $\nabla$ qiaotong@njglyy.com )

Nanjing Drum Tower Hospital

LEI WANG

Nanjing University

\section{Research Article}

Keywords: oridonin, low shear stress, atherosclerosis, endothelial cell dysfunction, oxidative stress, zebrafish, nuclear factor erythroid 2-related factor 2

Posted Date: July 12th, 2021

DOI: https://doi.org/10.21203/rs.3.rs-640059/v1

License: (c) (i) This work is licensed under a Creative Commons Attribution 4.0 International License. Read Full License 


\section{Abstract \\ Background}

Atherosclerosis (AS) is the primary cause of cardiovascular disease and the incidence is extremely common; however, there are currently few drugs that can effectively treat AS. Although oridonin has been widely used to treat inflammation and cancer for numerous years, to the best of our knowledge, its protective effect against AS has not been reported. Therefore, the present study aimed to investigate whether oridonin attenuated AS.

\section{Methods}

By using text mining, chemometric and chemogenomic methods, oridonin was predicted to be a beneficial agent for the treatment of AS. A parallel flow chamber was used to establish a low shear stress (LSS)-induced endothelial cell (EC) dysfunction model. Briefly, ECs were exposed to $3 \mathrm{dyn} / \mathrm{cm}^{2}$ LSS for 30 min and subsequently treated with oridonin or transfected with a small interfering RNA (siRNA) targeting nuclear factor erythroid 2-related factor 2 (NRF2). Reactive oxygen species (ROS), superoxide dismutase (SOD), malondialdehyde (MDA), glutathione (GSH) and glutathione disulfide (GSSG) in EA.hy926 cells were analyzed to determine the level of oxidative stress. The nitric oxide (NO) levels and mRNA expression levels of endothelial NO synthase (eNOS), endothelin-1 (ET-1) and prostaglandin synthase (PGIS) in EA.hy926 cells were analyzed to determine EC dysfunction. Furthermore, the mRNA expression levels of NRF2 were analyzed using reverse transcription-quantitative PCR. In addition, zebrafish were fed with a high-cholesterol diet to establish a zebrafish AS model, which was used to observe lipid accumulation and inflammation under a fluorescence microscope.

\section{Results}

We found LSS led to oxidative stress and EC dysfunction; this was primarily indicated through the significantly decreased SOD and GSH content, the significantly increased MDA, GSSG and ROS content, the upregulated mRNA expression levels of ET-1, and the downregulated NO levels and mRNA expression levels of eNOS and PGIS in ECs. Notably, oridonin could improve LSS-induced oxidative stress and EC dysfunction, and the effects of oridonin were reversed by the transfection with NRF2 siRNA. Oridonin also attenuated lipid accumulation and neutrophil recruitment at the LSS regions in the zebrafish AS model.

\section{Conclusions}

In conclusion, the results of the present study suggested that oridonin may ameliorate LSS-induced EC dysfunction and oxidative stress by activating NRF2, thereby attenuating AS. 


\section{Introduction}

Atherosclerosis (AS) is characterized by endothelial dysfunction, inflammation, progressive lipid deposition and vessel stiffness, which is accompanied by potential complications such as myocardial infarction or stroke $(1,2)$. Previous studies have demonstrated that atherosclerotic coronary artery disease is the main cause of cardiovascular disease mortality $(3,4)$. Thus, it remains a priority to identify an effective therapeutic method or drug to attenuate or cure AS.

Recently, network pharmacology has been proposed as a novel discipline to investigate the complex interactions of compounds and biology. The potential targets of compounds are identified on a proteomewide scale with computational ligand-based target prediction methods (5). Multiple studies have confirmed that combining computational and experimental methods can effectively discover novel targets for drugs $(6,7)$.

Oridonin, the major active ingredient of the traditional Chinese medicinal herb Rabdosia rubescens, was proven to possess antitumor activity, and it has been used to treat liver, esophageal and pancreatic cancer in the clinic (8-11). Previous studies have also reported that oridonin had anti-inflammatory effects and that it could inhibit the proliferation of endothelial cells (ECs), which are closely related to the pathogenesis of AS $(12,13)$. However, to the best of our knowledge, its protective effect against AS has not been reported. In the present study, the molecular mechanisms underlying the multi-target effects of oridonin in AS were determined. Based on critical text mining, and chemometric and chemogenomic methods, it was hypothesized that the key targets of oridonin in AS may include adenylate cyclase type1 (ADCY1), ADCY2, nuclear factor erythroid 2-related factor 2 (NRF2) and nuclear receptor subfamily 0 group B member 1 (NROB1).

Although AS has numerous systemic risk factors, such as hypertension, obesity and smoking, it preferentially develops near branches and bends in blood vessels, where the ECs are exposed to oscillatory or low shear stress (LSS) (14). Since this discovery, SS has been illustrated to serve an important role in the occurrence of AS (15). LSS was discovered to induce oxidative stress and EC dysfunction, including reducing the levels of nitric oxide (NO) and upregulating endothelin-1 (ET-1) expression levels, which eventually lead to plaque formation. However, the mechanism for the conversion of mechanical signals to biological signals in ECs is only partially understood. A few detectors of LSS have been proposed, including mechanosensitive transcription factors (MSTFs), which may also be promising therapeutic targets for the treatment of endothelial dysfunction or AS. NRF2 is a MSTF that can sense the blood flow and regulate EC physiology (16). Therefore, the present study selected NRF2, as a potential target of oridonin, for further investigations.

The current study used both a LSS-induced EC dysfunction model and a high-cholesterol diet (HCD)induced zebrafish AS model to verify the target, which was identified previously. The findings revealed that oridonin ameliorated LSS-induced EC dysfunction by activating NRF2, thereby attenuating AS. 


\section{Materials And Methods}

Test compounds, chemicals and reagents. Oridonin was purchased from Selleck Chemicals. MTT reagent was purchased from Sigma-Aldrich; Merck KGaA. The other chemicals and reagents were of analytical grade.

Target identification. To identify the targets of oridonin and determine compound-target interaction profiles, an in silico approach was applied as described in our previous study (17); this approach integrated text mining with chemometric and chemogenomic methods. In the present study, text mining was first used to identify the targets of oridonin; this was performed using the Traditional Chinese Medicine Systems Pharmacology Database and Analysis Platform ( https://tcmspw.com/tcmsp.php), a database comprised of systems pharmacology data for drug discovery related to herbal medicines (18). Subsequently, the virtual chemical fingerprint similarity ensemble approach method (http://sea.bkslab.org) was used to predict potential targets of oridonin. Finally, the potential target proteins identified were further subjected to The Pharmacogenomics Knowledge Base (http://www.pharmgkb.org), and Comparative Toxicogenomics Database (http://ctdbase.org) to remove noise and errors. In addition, this enabled an accurate view of oridonin targets for cardiovascular diseases, including ADCY1, ADCY2, NRF2 and NROB1 (Table I). It was previously reported that NRF2 served an important role in LSS-induced EC dysfunction and AS $(16,20)$, while another previous study discovered that oridonin improved inflammation and oxidative stress by activating NRF2 (21). Therefore, NRF2 was selected for further investigations.

Human umbilical vein EC (HUVEC) culture and viability assay. The HUVEC cell line, EA.hy926, was obtained from the American Type Culture Collection. EA.hy926 cells were cultured in DMEM (Invitrogen; Thermo Fisher Scientific, Inc.) supplemented with 10\% FBS, $100 \mathrm{U} / \mathrm{ml}$ penicillin and $100 \mu \mathrm{g} / \mathrm{ml}$ streptomycin, and maintained at $37^{\circ} \mathrm{C}$ in a $5 \% \mathrm{CO}_{2}$ humified incubator. Upon the EA.hy 926 cells reaching the logarithmic growth phase, the cells were treated with $0.1 \%$ DMSO or various concentrations of oridonin $(0,25,50,100,200$ or $400 \mu \mathrm{g} / \mathrm{ml})$ for $24 \mathrm{~h}$. The cell viability was determined using an MTT assay, with $\geq 3$ independent experiments performed in triplicate.

LSS experiment. Upon EA.hy926 cells reaching the logarithmic growth phase, the cells were seeded onto a glass slide $(30 \times 50 \mathrm{~mm})$ and treated with $0.1 \%$ DMSO, $100 \mu \mathrm{g} / \mathrm{ml}$ oridonin or $100 \mu \mathrm{g} / \mathrm{ml}$ oridonin combined with the transfection of small interfering RNA (siRNA) targeting NRF2 $(0.1 \mu \mathrm{mol} / \mathrm{l})$ for $24 \mathrm{~h}$. Following treatment for $24 \mathrm{~h}$, the LSS test was performed. Briefly, a parallel flow chamber ( Shanghai Medical Equipment Works Co., Ltd), which consists of two stainless steel plates and a silicone gasket, was used in the present study. The glass slide with confluent cells was placed on the lower plate of the chamber and then subjected to LSS induced by continuous fluid flow. SS values were modulated by the flow through the chamber.

EC dysfunction assay. The NO levels, and ET-1, endothelial NO synthase (eNOS) and PGIS mRNA expression levels in EA.hy926 cells were analyzed to determine EC dysfunction. Following the LSS 
experiment, EA.hy 926 cells were incubated with $50 \mu \mathrm{M}$ NO-specific fluorescent dye, 4-Amino-5Methylamino-2',7'-Difluorofluorescein diacetate (DAF-FM diacetate; Beyotime Institute of Biotechnology). Following the incubation, the EA.hy926 cells were washed twice with PBS and then visualized and analyzed via fluorescence microscopy. The fluorescence intensity was semi-quantified from $\geq 3$ random fields of view per slide from three different slides.

The mRNA expression levels of ET-1, eNOS and PGIS in EA.hy926 cells were analyzed using reverse transcription-quantitative PCR (RT-qPCR). Briefly, following the LSS experiment, total RNA was extracted from the cells using TRIzol ${ }^{\circledR}$ reagent (Invitrogen; Thermo Fisher Scientific, Inc.), according to the manufacturer's protocol. Total RNA was reverse transcribed into cDNA using the PrimeScript ${ }^{\text {TM }}$ RT Master mix (Perfect Real-Time) (Takara Bio, Inc.), according to the manufacturer's protocol. The resultant cDNA was used as a template for qPCR analysis in the Thermal Cycler Dice® Real-Time system (Takara Bio, Inc.). The primers used for the qPCR were designed by Primer3 software and are listed in Table II. The mRNA expression data are expressed as the relative expression ratio normalized to GAPDH.

Oxidative stress assay. Following the LSS experiment, EA.hy926 cells were collected to measure secreted superoxide dismutase (SOD) activity, malondialdehyde (MDA) content, glutathione (GSH) content and glutathione disulfide (GSSG) content using ELISAs (Beijing Solarbio Science \& Technology Co., Ltd.), according to the manufacturers' protocols.

Following the LSS experiment, EA.hy 926 cells were also incubated with the ROS-specific fluorescent dye dihydroethidium (DHE; $50 \mu \mathrm{M}$; Beyotime Institute of Biotechnology). Following the incubation, the EA.hy 926 cells were washed in PBS twice and then visualized and analyzed via fluorescence microscopy. The fluorescence intensity was semi-quantified from $\geq 3$ random fields of view per slide from three different slides.

Zebrafish AS model. Zebrafish lines used in this research were purchased from China Zebrafish Resource Center (CZRC, China). All studies involving zebrafish manipulations were approved by the institutional animal use and care committee of Nanjing Drum Tower Hospital. All animal experiments were performed in accordance with the National Institutes of Health Guidelines for the Care and Use of Laboratory Animals. About the euthanasia of zebrafish, we put zebrafish larvae in a $2 \mathrm{~g} / \mathrm{L}$ tricaine solution for about 1 minute for euthanasia, meanwhile we use the lost of righting reflex, opercular movements and the silence of heart beating to confirm death. The accumulation of lipids in zebrafish blood vessels was detected to reflect early atherosclerotic plaque formation. As determined from a previous study (22), zebrafish larvae were fed with a HCD to establish a zebrafish AS model. In total, 5 days post-fertilization (dpf) $T g$ (fli1:EGFP) zebrafish larvae, constitutively expressing GFP in the ECs, were fed for 10 days with the HCD enriched with $4 \%$ cholesterol and supplemented with $10 \mu \mathrm{g} / \mathrm{g}$ fluorescent cholesteryl ester analog. A large amount of red fluorescent lipid accumulation in the zebrafish green blood vessels could be observed under a fluorescence microscope.

Detection of the effect of oridonin treatment on early AS plaque formation. The $5 \mathrm{dpf} \operatorname{Tg}($ fli1:EGFP) zebrafish larvae were randomly divided into five groups: i) Control group; ii) AS group; iii) $1 \mathrm{mg} / \mathrm{l}$ oridonin 
group; iv) $50 \mathrm{mg} / \mathrm{l}$ oridonin group; and v) $100 \mathrm{mg} / \mathrm{l}$ oridonin group. In the control group, the larvae were fed with $10 \mu \mathrm{g} / \mathrm{g}$ fluorescent cholesteryl ester analog for 10 days. In the AS group, the larvae were fed for 10 days with the HCD enriched with $4 \%$ cholesterol, and supplemented with $10 \mu \mathrm{g} / \mathrm{g}$ fluorescent cholesteryl ester analog. In the various concentrations of oridonin (1,50 and $100 \mathrm{mg} / \mathrm{l})$ treatment groups, the larvae were fed for 10 days with the HCD enriched with $4 \%$ cholesterol, and supplemented with 10 $\mu \mathrm{g} / \mathrm{g}$ fluorescent cholesteryl ester analog. At the same time, the larvae were treated with various concentrations of oridonin (1,50 and $100 \mathrm{mg} / \mathrm{l})$. Images of the caudal vasculature in the live larvae revealed that the vasculature of the control and HCD larvae were stained diffusely red, with bright fluorescent lipid deposits in the blood vessel wall visible in the HCD larvae. A study previously reported that these accumulated lipids are similar to the plaques observed during early AS (23).

Detecting inflammation in the zebrafish AS model. The presence of inflammation in $\operatorname{Tg}(m p x$ :EGFP) zebrafish was subsequently observed through specifically labeling the neutrophils with GFP. In the control group, $5 \mathrm{dpf} \operatorname{Tg}(m p x: E G F P)$ zebrafish larvae were fed with conventional feed for 10 days. In the AS group, $5 \mathrm{dpf} \operatorname{Tg}(m p x: E G F P)$ zebrafish larvae were fed for 10 days with a HCD enriched with $4 \%$ cholesterol. In the various concentrations of oridonin (1,50 and $100 \mathrm{mg} / \mathrm{l})$ treatment groups, $5 \mathrm{dpf} \mathrm{Tg}(\mathrm{mpx}$ :EGFP) zebrafish larvae were fed for 10 days with the HCD enriched with $4 \%$ cholesterol, and treated with various concentrations of oridonin $(1,50$ and $100 \mathrm{mg} / \mathrm{l})$. The quantity and recruitment of neutrophils was observed under a fluorescence microscope to determine the levels of inflammation in each group of zebrafish.

Determining the effect of oridonin treatment on the lipid levels in the zebrafish AS model. The $5 \mathrm{dpf}$ wildtype AB-line zebrafish larvae were randomly divided into five groups as said above. In the control group, zebrafish larvae were fed with normal basal feed (which did not contain $4 \%$ cholesterol) for 10 days. In the AS group, zebrafish larvae were fed a HCD enriched with $4 \%$ cholesterol for 10 days. In the various concentrations of oridonin $(1,50$ and $100 \mathrm{mg} / \mathrm{l})$ treatment groups, zebrafish larvae were fed for 10 days with an HCD enriched with $4 \%$ cholesterol and treated with various concentrations of oridonin $(1,50$ and $100 \mathrm{mg} / \mathrm{l}$ ). After 10 days of feeding and $24 \mathrm{~h}$ of fasting, Nile Red staining was used to detect the lipid levels in each group of zebrafish. The stock solution $(1.25 \mathrm{mg} / \mathrm{ml}$ ) of Nile Red (cat. no. N-1142; Invitrogen; Thermo Fisher Scientific, Inc.) was prepared in acetone and stored in the dark at $-20^{\circ} \mathrm{C}$. For the staining of the zebrafish, the stock solution was diluted to $50 \mathrm{ng} / \mathrm{ml}$ in egg water and incubated for $15 \mathrm{~min}$ at $28^{\circ} \mathrm{C}$ in the dark. The fishes were washed with distilled water 3 times and anesthetized with a few drops of tricaine solution $(0.2 \mathrm{mg} / \mathrm{ml}$; pH 7.0; Sigma-Aldrich; Merck KGaA). The zebrafish were subsequently mounted in $4 \%$ methylcellulose and the extent of Nile Red staining was imaged using an Olympus SZX16 microscope (Olympus Corporation), which was used for yellow fluorescent imaging.

Detection of the effect of oridonin on the oxidative stress in the zebrafish AS model. The $5 \mathrm{dpf}$ wild-type AB-line zebrafish larvae were randomly divided into five groups as said above. The description of each group of zebrafish is consistent with that described in the section describing the detection of the lipid levels in the zebrafish AS model. After 10 days of feeding and $24 \mathrm{~h}$ of fasting, dichloro-dihydrofluorescein diacetate was used to detect the levels of ROS in each group of zebrafish. 
Biochemical measurements. The $5 \mathrm{dpf}$ wild-type AB-line zebrafish larvae were randomly divided into five groups as said above. The description of each group of zebrafish is consistent with that described in the section describing the detection of the lipid levels in the zebrafish AS model. After 10 days of feeding and $24 \mathrm{~h}$ of fasting, 5 larvae from each larva in each group were randomly selected and sacrificed as one sample, and six samples were prepared for testing each index. Triglyceride (TG) levels, total cholesterol (TC) levels, SOD activity and MDA levels were measured using commercial assay kits (Jiancheng Bioengineering Institute, Nanjing, China,http://www.njjcbio.com/), according to the manufacturers' protocols. The results of the aforementioned assays were quantified using a multifunctional microplate reader.

Statistical analysis. Statistical analyses were performed using SPSS 22.0 software (IBM Corp.) and data are presented as the mean \pm standard deviation. Statistical differences between groups were determined using an ANOVA with Bonferroni adjustment for multiple comparisons. $\mathrm{P}<0.05$ was considered to indicate a statistically significant difference.

\section{Results}

Target screening. Through combining text mining and chemogenomic prediction methods, 4 potential protein targets for oridonin were obtained (Table I). It was previously discovered that NRF2 activation induced by high/laminar SS was important for EC adaptation to oxidative stress, in addition to exerting anti-inflammatory roles (24). Furthermore, a previous study demonstrated that oridonin treatment improved inflammatory and oxidative stress by activating NRF2 (21). The present study used RT-qPCR to determine the effect of oridonin treatment on the expression levels of NRF2 and its downstream target genes, heme oxygenase 1 (HMOX1) and $\mathrm{NAD}(\mathrm{P}) \mathrm{H}$ dehydrogenase [quinone] 1 (NQ01). The results revealed that oridonin treatment significantly upregulated the mRNA expression levels of NRF2, HMOX1 and NQ01 in EA.hy926 cells (Fig. 1A-C). Therefore, NRF2 was further investigated in the present study.

Oridonin improves LSS-induced EC dysfunction by activating NRF2. EA.hy926 cells were treated with various concentrations of oridonin $(0,25,50,100,200$ or $400 \mu \mathrm{g} / \mathrm{ml})$ for $24 \mathrm{~h}$, and the cell viability was determined using an MTT assay at $24 \mathrm{~h}$. As shown in Fig. 2A, low doses of oridonin $(0-100 \mu \mathrm{g} / \mathrm{ml})$ did not inhibit cell proliferation, while higher doses of oridonin $(200-400 \mu \mathrm{g} / \mathrm{ml})$ inhibited cell proliferation. The half maximal inhibitory concentration value of oridonin was $337.84 \mu \mathrm{g} / \mathrm{ml}$ at $24 \mathrm{~h}$. Based on these results, $100 \mu \mathrm{g} / \mathrm{ml}$ oridonin was used in further studies.

Similar to our previous study (25), EA.hy 926 cells were exposed to laminar flow with a value of 0 or 3 $\mathrm{dyn} / \mathrm{cm}^{2}$ for $30 \mathrm{~min}$. A siRNA sequence targeting NRF2 was used to silence its expression, which was subsequently verified by RT-qPCR (Fig. 2B and Fig. S1). The intracellular NO activity was analyzed using the fluorescent probe DAF-FM diacetate; as shown in Fig. 2C, LSS significantly reduced the NO activity, while the treatment with $100 \mu \mathrm{g} / \mathrm{ml}$ oridonin significantly increased NO activity. Notably, the genetic knockdown of NRF2 counteracted the effects of oridonin on the intracellular NO activity. The expression levels of EC dysfunction-related genes (ET-1, eNOS and PGIS) were also determined using RT-qPCR. As 
shown in Fig. 2D-F, LSS significantly downregulated the mRNA expression levels of eNOS and PGIS, while significantly upregulating the ET-1 mRNA expression levels. These changes in the expression levels were significantly inhibited by $100 \mu \mathrm{g} / \mathrm{ml}$ oridonin, whereas the genetic silencing of NRF2 counteracted the effects of oridonin treatment on the mRNA expression levels of ET-1, eNOS and PGIS.

Oridonin improves LSS-induced oxidative stress by activating NRF2. The intracellular ROS activity was investigated using the fluorescent probe dye DHE. LSS significantly induced ROS activity, while the ROS activity was significantly inhibited by $100 \mu \mathrm{g} / \mathrm{ml}$ oridonin (Fig. 3A). Conversely, the genetic knockdown of NRF2 counteracted the effects of oridonin treatment on the activity of ROS. The levels of SOD, MDA, GSSG and GSH in EA.hy926 cells were also investigated. As shown in Fig. 3B-E, LSS significantly reduced SOD and GSH levels and significantly increased MDA and GSSG levels, while the effects of LSS were significantly inhibited by $100 \mu \mathrm{g} / \mathrm{ml}$ oridonin treatment. Meanwhile, the genetic silencing of NRF2 counteracted the effects of oridonin treatment on the levels of these oxidases.

Oridonin reduces the formation of early plaques in a zebrafish AS model. Severe plaques formed in the blood vessels of the zebrafish fed the HCD (Fig. 4), whereas the treatment with 50 and $100 \mu \mathrm{g} / \mathrm{ml}$ oridonin appeared to decrease the HCD-induced plaque formation. However, the treatment with $1 \mu \mathrm{g} / \mathrm{ml}$ oridonin had not significant effect on HCD-induced plaque formation.

Oridonin reduces lipid accumulation in a zebrafish AS model. The lipid levels in zebrafish were detected using Nile Red staining. As shown in Fig. 5A, compared with the control group, the lipid levels were significantly increased in the AS group. However, compared with the AS group, the treatment with 50 or $100 \mu \mathrm{g} / \mathrm{ml}$ oridonin appeared to decrease the lipid levels, while $1 \mathrm{mg} / \mathrm{l}$ oridonin had no significant effect on the lipid levels. A similar result was also obtained by measuring the TG and TC contents in the zebrafish; compared with the control group, the levels of TGs and TC were significantly increased in the AS group (Fig. 5B and C). In contrast, compared with the AS group, the treatment with 50 or $100 \mu \mathrm{g} / \mathrm{ml}$ oridonin significantly decreased the levels of TGs and TC, while $1 \mathrm{mg} / \mathrm{l}$ oridonin did not markedly decrease the levels of TGs and TC.

Oridonin reduces oxidative stress in a zebrafish AS model. As shown in Fig. 6A, compared with the control group, the ROS levels were significantly increased in the AS group. Conversely, compared with the AS group, the treatment with 50 or $100 \mu \mathrm{g} / \mathrm{ml}$ oridonin appeared to decrease the ROS levels, while $1 \mathrm{mg} / \mathrm{l}$ oridonin did not affect the ROS levels. Consistent results were obtained by measuring the SOD activity and MDA content in the zebrafish. That is, compared with the control group, the SOD activity was significantly decreased, while the MDA content was significantly increased, in the AS group (Fig. 6B and C). Meanwhile, compared with the AS group, the treatment with 50 and $100 \mu \mathrm{g} / \mathrm{ml}$ oridonin significantly reduced the MDA content and significantly increased SOD activity. Similar to the previous findings, $1 \mathrm{mg} / \mathrm{l}$ oridonin had no significant effect on the SOD activity and MDA content.

Oridonin reduces inflammation in a zebrafish AS model. A large number of neutrophils were identified in the blood vessels of the zebrafish fed the HCD, while the treatment with 50 or $100 \mu \mathrm{g} / \mathrm{ml}$ oridonin appeared to decrease the HCD-induced increase in neutrophils (Fig. 7). However, the treatment with 1 
$\mu \mathrm{g} / \mathrm{ml}$ oridonin did not significantly inhibit the HCD-induced increase in the number of neutrophils in the blood vessels.

\section{Discussion}

The present study used a novel systems pharmacology method to identify new drugs for the potential treatment of cardiovascular diseases. The hubs and the centric elements of the network were analyzed to identify key targets, and through this analysis, 4 targets with high degrees were identified, namely ADCY1, ADCY2, NRF2 and NROB1. Out of these targets, NRF2 was selected for further investigations and it was discovered that oridonin treatment significantly upregulated the mRNA expression levels of NRF2, and its target genes, such as HMOX1 and NQ01, in EA.hy926 cells. In addition, the atheroprotective effects of oridonin on ECs were weakened following the genetic silencing of NRF2.

LSS has been reported to serve an important role in the occurrence of EC dysfunction and AS (26). ECs, the innermost monolayer of the vessel wall, are the earliest cells to encounter SS; thus, they are the major cell type to sense the hemodynamic forces from the blood flow and transfer the mechanical signals to biochemical signals to regulate various signaling pathways. Therefore, improving EC dysfunction induced by LSS is hypothesized to be an effective way to prevent or cure AS. EC dysfunction is characterized by an imbalance between vasodilatory and vasoconstrictive molecules; these molecules, including prostacyclin $\left(\mathrm{PGI}_{2}\right), \mathrm{NO}$ and $\mathrm{ET}-1$, are synthesized and released by $\mathrm{ECs}$. $\mathrm{PGI}_{2}$ and $\mathrm{NO}$ are effective vasodilators and are produced by PGIS and eNOS, respectively, while ET-1 is a potent vasoconstrictor (27). In the present study, a parallel flow chamber was used to establish an LSS-induced EC dysfunction in vitro model and it was subsequently discovered that LSS significantly reduced the activity of NO. In addition, LSS significantly downregulated the mRNA expression levels of eNOS and PGIS, while significantly upregulating ET-1 mRNA expression levels.

LSS was previously found to increase oxidative stress levels in ECs (25). The findings of the present study illustrated that LSS significantly reduced the activity of SOD and GSH, while significantly increasing the levels of ROS, MDA and GSSG. Notably, oridonin treatment improved the LSS-induced EC dysfunction, while the genetic silencing of NRF2 counteracted the effects of oridonin treatment on LSS-induced EC dysfunction. These results suggested that oridonin may improve LSS-induced EC dysfunction by activating NRF2.

NRF2 is an important mechanosensitive transcription factor that regulates the expression of a group of genes responsible for inflammation and oxidative stress. Thus, it is considered to have a close relationship with EC dysfunction and AS. In the high/laminar SS regions of the vasculature, NRF2 was discovered to be activated, where it participated in the adaptative mechanism of the ECs to oxidative and inflammatory stress (16). In the current study, the expression levels of NRF2 were downregulated by LSS. Thus, identifying a drug targeting the downregulation of NRF2 in the LSS regions may be the future of AS treatment. 
To investigate whether oridonin treatment attenuated AS, zebrafish were fed a HCD to establish a zebrafish AS model. The zebrafish is a unique vertebrate model that combines the advantageous characteristics of invertebrate models (small size, powerful genetic tractability, high fecundity, ease of maintenance and relatively low costs) with a high degree of evolutionary conservation with mammals. Thus, zebrafish are invaluable, not only for studying vertebrate development and physiology, but also for modeling human diseases (28). Zebrafish are also used to study AS, in which the hypercholesterolemia zebrafish is currently the most commonly used zebrafish AS model (22). In the present study, severe lipid accumulation occurred in the blood vessels of the zebrafish fed a HCD, and studies have previously shown that this accumulation of lipids in the blood vessels

is similar to the early plaques of AS(23). These results further suggested that HCD-fed zebrafish larvae may be used to study human AS. A recent study illustrated that the arteriovenous differentiation was incomplete during the larval stage of zebrafish, thus the veins and arteries are considered to be similar during this period. The only difference is that the blood flow in the veins is much slower, which signifies that the wall SS induced by the blood flow is lower in the veins, especially in those near the tail (29). This hypothesis is consistent with the results obtained in the present study, as the lipid accumulation, which was represented by red fluorescence, was more severe in the veins near the tail. However, the results revealed that oridonin treatment could significantly reduce the accumulation of lipids in the blood vessels of a zebrafish AS model. These findings indicated that oridonin may exert a beneficial therapeutic effect on early atherosclerotic plaque formation caused by LSS.

It is generally considered that hyperlipidemia, inflammation and oxidative stress lead to the initiation and development of AS (30-32). In the present study, a zebrafish AS model was established by feeding zebrafish with a HCD for 10 days. Zebrafish in the AS group demonstrated signs of hyperlipidemia, oxidative stress and inflammation, as expected. These results further illustrated that zebrafish can be used to study AS. Notably, oridonin treatment significantly improved the HCD-induced hyperlipemia, oxidative stress and inflammation. These findings also indicated that the anti-AS effect of oridonin may be related to its lipid-lowering, antioxidant and anti-inflammatory effects.

There are many limitations in this study, we revealed the potential of oridonin in the treatment of atherosclerosis, and briefly studied the role of NRF2 in the treatment of atherosclerosis. The mechanism of our research is not deep enough and we lack the protein expression analysis. A fluorescence quantification by flow cytometry will be a good supplementary result to show the changes between intracellular NO activity and we will supplement it in later experiments. Most importantly, we only found that oridonin can affect the expression of NRF2, but whether NRF2 is the target of oridonin needs more research in the future.

In conclusion, the findings of the present study revealed that oridonin exerted effects in LSS-induced EC dysfunction and HCD-induced hyperlipidemia, inflammation and oxidative stress, and inhibited plaque formation in AS. Therefore, in the future, oridonin may be considered as a potential therapeutic option for AS. 


\section{List Of Abbreviations}




\begin{tabular}{|ll|}
\hline AS & Atherosclerosis \\
\hline EC & Endothelial cell \\
\hline LSS & Low shear stress \\
\hline SS & Shear stress \\
\hline NRF2 & Nuclear factor erythroid 2-related factor 2 \\
\hline HMOX1 & NAD(P)H:quinoneoxidoreductaseNQ01: \\
\hline ADCY1 & Adenylate cyclase 1 \\
\hline ADCY2 & Adenylate cyclase 2 \\
\hline MTT & Thiazolyl blue \\
\hline RT-qPCR & Realtime quantitative PCR \\
\hline SiRNA & Small interfering RNA \\
\hline ROS & Reactive oxygen species \\
\hline LDL & Low-density lipoprotein \\
\hline Ox-LDL & Oxidized Low-density lipoprotein \\
\hline NF-KB & Nuclear factor kappa-B \\
\hline HIF-1a & Hypoxia inducible factor-1-alpha \\
\hline IL-6 & Interleukin-6 \\
\hline TNF-a & Tumor necrosis factor-a \\
\hline MSTFs & Mechanosensitive transcription factors \\
\hline NADPH & Nicotinamide adenine dinucleotide phosphate \\
\hline SOD & Superoxide dismutase \\
\hline MDA & Malondialdehyde \\
\hline GSH & Glutathione \\
\hline GSSG & Glutathione (Oxidized) \\
\hline HCD & Carboxymethylcellulose sodium \\
\hline High-cholesterol diet \\
\hline Dimethyl sulfoxide \\
\hline H
\end{tabular}

Page 12/19 


\begin{tabular}{|ll|}
\hline AS & Atherosclerosis \\
\hline DMEM & Dulbecco's modified Eagle's medium \\
\hline DEPC & Diethy pyrocarbonate \\
\hline MAPK & Mitogen-activated protein kinase \\
\hline NO & Nitric oxide \\
\hline PBS & Phosphate buffered saline \\
\hline FBS & Fetal calf serum \\
\hline SDS & Sodium dodecylsulphate \\
\hline MCP-1 & Monocyte chemotactic protein-1 \\
\hline ICAM-1 & Intercellular cell adhesion molecule-1 \\
\hline VCAM-1 & vascular cell adhesion molecule-1 \\
\hline VWF & Von willebrand factor \\
\hline ET-1 & Endothelin - 1 \\
\hline PGIS & Prostacyclin synthase \\
\hline PGI2 & Prostaglandin l-2 \\
\hline MS-222 & Tricaine \\
\hline
\end{tabular}

\section{Declarations}

\section{Ethics approval and consent to participate}

All studies involving zebrafish manipulations were approved by the institutional animal use and care committee of Nanjing Drum Tower Hospital. All animal experiments were performed in accordance with the National Institutes of Health Guidelines for the Care and Use of Laboratory Animals. My colleagues and I have confirmed that this study was carried out in compliance with the ARRIVE guidelines.

\section{Consent for publication}

Not applicable

\section{Availability of data and materials}

All data generated or analysed during this study are included in this published article [and its supplementary information files]

\section{Competing interests}


The authors declare no conflict of financial and non-financial interests.

\section{Funding}

This study was supported by the National Natural Science Foundation of China (Grant numbers 81870348 81370387$)$, Nanjing Key Medical Science and Technology Development Project (Grant number ZKX19019).

\section{Authors' Contributions}

$\mathrm{ZC}$ and $\mathrm{HL}$ contributed equally to the work. $\mathrm{ZC}$ and $\mathrm{HL}$ conceived and designed the experiments and wrote the paper. SUBINUR, CL, YL and LW provided technical support. JC and TQ supervised the whole project.

\section{Acknowledgments}

This work was supported by Nanjing Medical University and Nanjing drum tower hospital. This study was supported by the National Natural Science Foundation (81870348®81370387)

\section{References}

Citation list not provided with this version

\section{Tables}

Table 1

The dock score of oridonin targets.

\begin{tabular}{|lll|}
\hline Gene name & Description & Dock score \\
\hline ADCY1 & Adenylate cyclase type 1 & 8.9359 \\
\hline ADCY2 & Adenylate cyclase type 2 & 15.5202 \\
\hline NRF2 & Nuclear factor erythroid 2-related factor 2 & 11.2796 \\
NROB1 & Nuclear receptor subfamily O group B member 1 & 10.8533 \\
\hline
\end{tabular}


Table 2

The primers used for real-time PCR.

\begin{tabular}{|llll|}
\hline Gene & Species & & Primer sequence $\left(\mathbf{5}^{\prime} \rightarrow \mathbf{3}^{\prime}\right)$ \\
\hline ET-1 & Homo & Forward & GGCTGAAGGATCGCTTTGAGA \\
\cline { 3 - 4 } eNOS & Homo & Foverse & GCTCAGCGCCTAAGACTGTTT \\
\cline { 3 - 4 } & & Reverse & GGTTTCCAGCCCTGCTGTAT \\
PGIS & Homo & Forward & ATTACAACATGCCCTGGGGG \\
& & Reverse & TGCGTTGATCAGCTCCAAGT \\
NRF2 & Homo & Forward & AGGTTGCCCACATTCCCAAA \\
\cline { 3 - 4 } & & Reverse & ACGTAGCCGAAGAAACCTCA \\
\hline HMOX1 & Homo & Forward & TAGAAGAGGCCAAGACTGCG \\
\cline { 3 - 4 } & & Reverse & GGGCAGAATCTTGCACTTTGTT \\
\hline NQ01 & Homo & Forward & GGTTTGGAGTCCCTGCCATT \\
\cline { 3 - 4 } & & Reverse & GCCTTCTTACTCCGGAAGGG \\
\hline GAPDH & Homo & Forward & CCATGGGGAAGGTGAAGGTC \\
\cline { 3 - 4 } & & Reverse & GCGCCCAATACGACCAAATC \\
\cline { 3 - 4 } & &
\end{tabular}

\section{Figures}
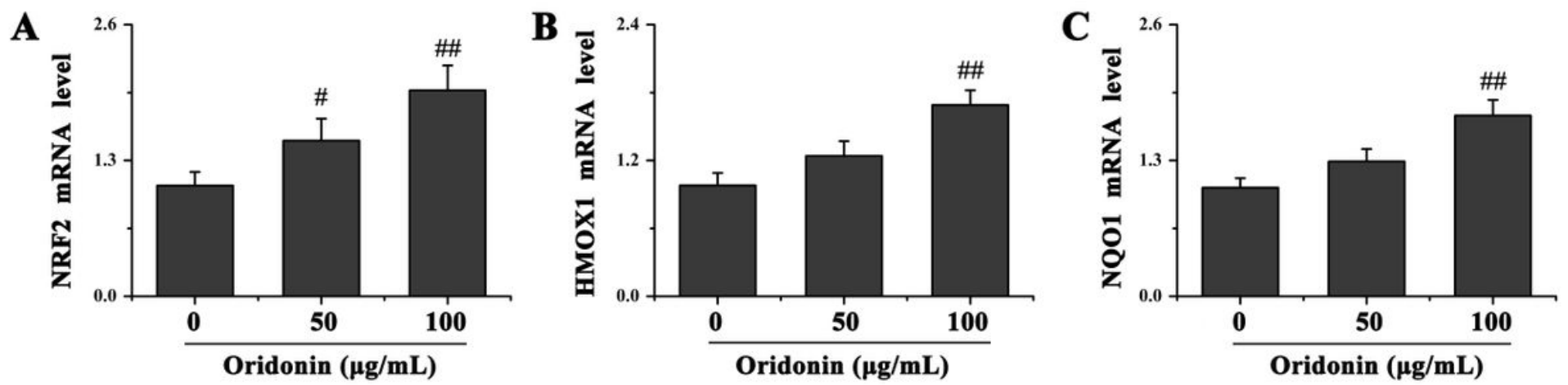

Figure 1

Oridonin upregulates the mRNA expression levels of (A) NRF2, (B) HMOX1 and (C) NQ01 in EA.hy926 cells. NRF2, nuclear factor erythroid 2-related factor 2; HMOX1, heme oxygenase 1; NQO1, NAD(P)H dehydrogenase [quinone] 1. 
$\mathbf{A}$

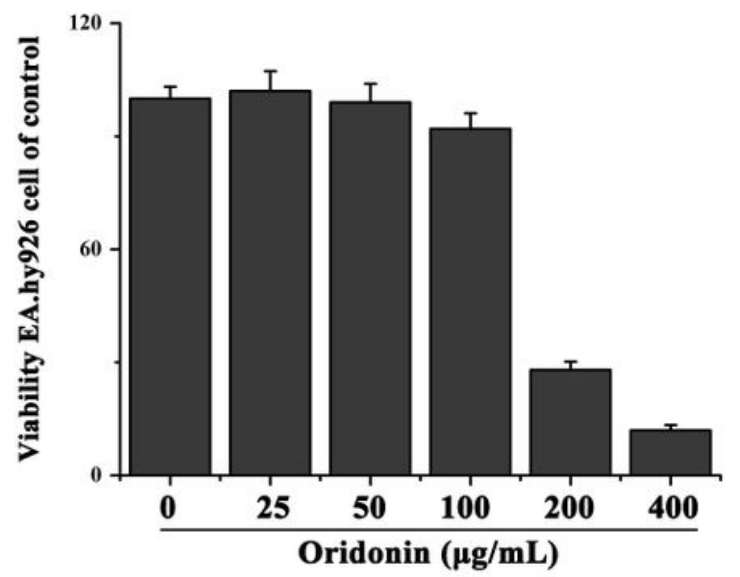

B

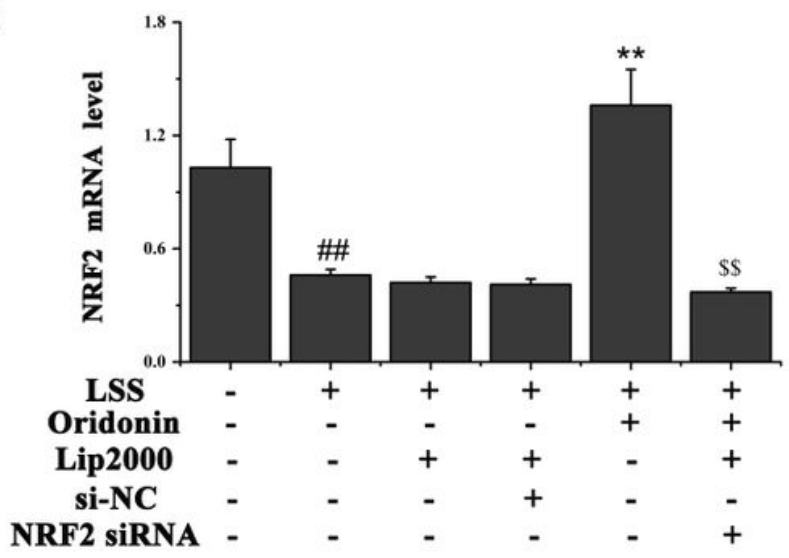

\section{C}

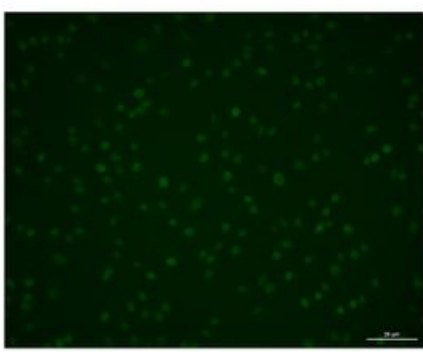

LSS

Oridonin NRF2 siRNA

D

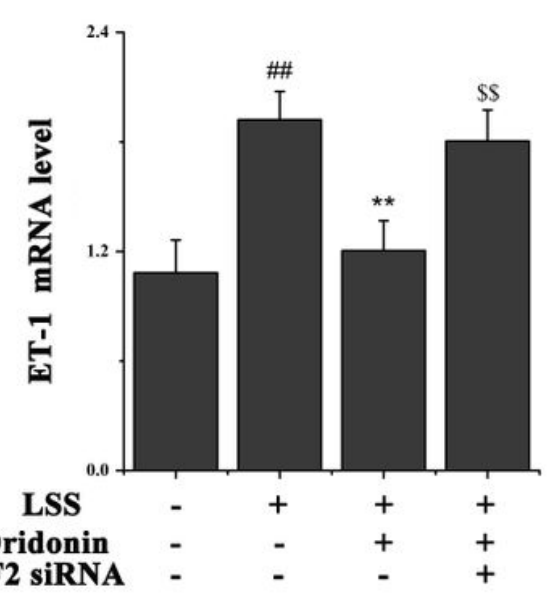

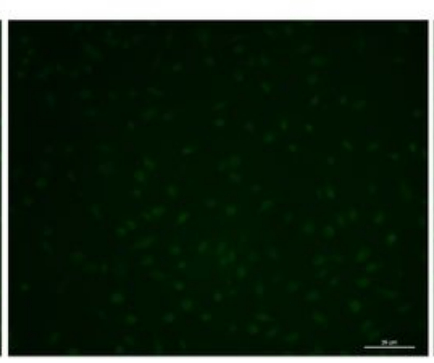

$+$

$\mathbf{E}$

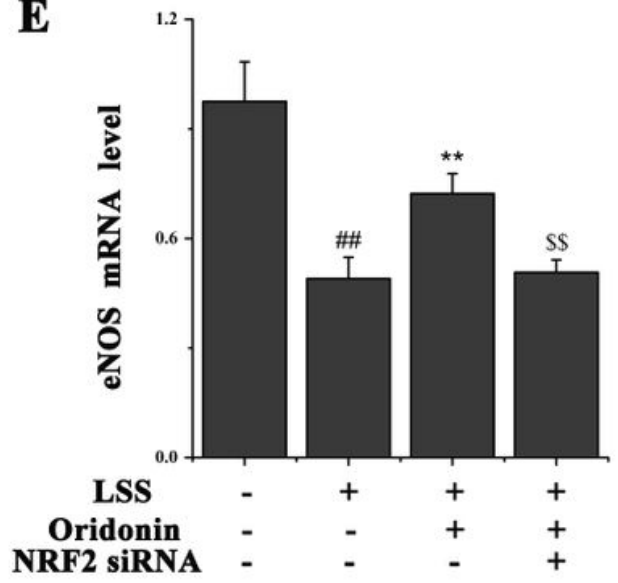

F

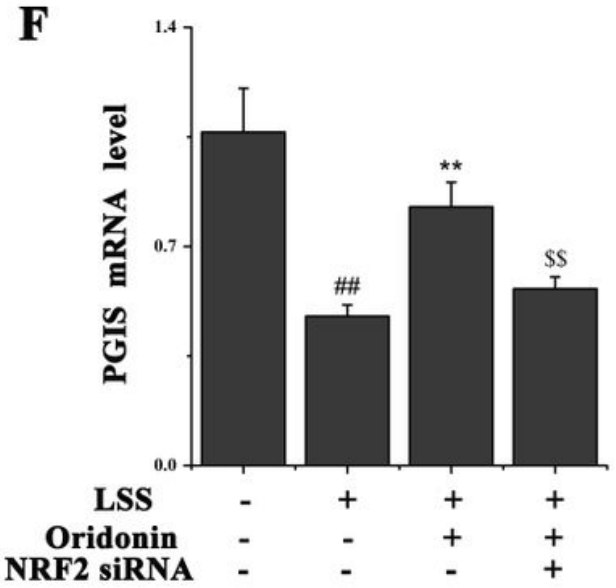

\section{Figure 2}

Oridonin improves LSS-induced EC dysfunction by activating NRF2. (A) Effect of oridonin on EA.hy926 cell viability. EA.hy 926 cells were treated with various concentrations of oridonin $(0-400 \mu \mathrm{g} / \mathrm{ml})$, and the viability was determined using an MTT assay at $24 \mathrm{~h}$. (B) Genetic silencing of NRF2 counteracted the effects of oridonin treatment on the mRNA expression levels of NRF2. (C) Results of the fluorescent probe DAF-FM diacetate revealed that oridonin treatment increasedthe LSS-induced reduced NO activity by activating NRF2. (D) Oridonin treatment decreased the LSS-induced upregulation in ET-1 mRNA expression levels. (E) Oridonin treatment increased the LSS-induced downregulation in eNOS mRNA expression levels. (F) Oridonin treatment increased the LSS-induced downregulation in PGIS mRNA expression levels. \#\#P<0.01 vs. LSS $(-)+$ oridonin $(-)+$ NRF2 siRNA $(-) ; * \star P<0.01$ vs. LSS $(+)+$ oridonin $(-)$ + NRF2 siRNA (-); \$\$P<0.01 vs. LSS (+) + oridonin (+) + NRF2 siRNA (-). LSS, low shear stress; NRF2, 
nuclear factor erythroid 2-related factor 2; EC, endothelial cell; ROS, reactive oxygen species; ET-1, endothelin-1; eNOS, endothelial nitric oxide synthase; PGIS, prostaglandin synthase; siRNA, small interfering RNA.

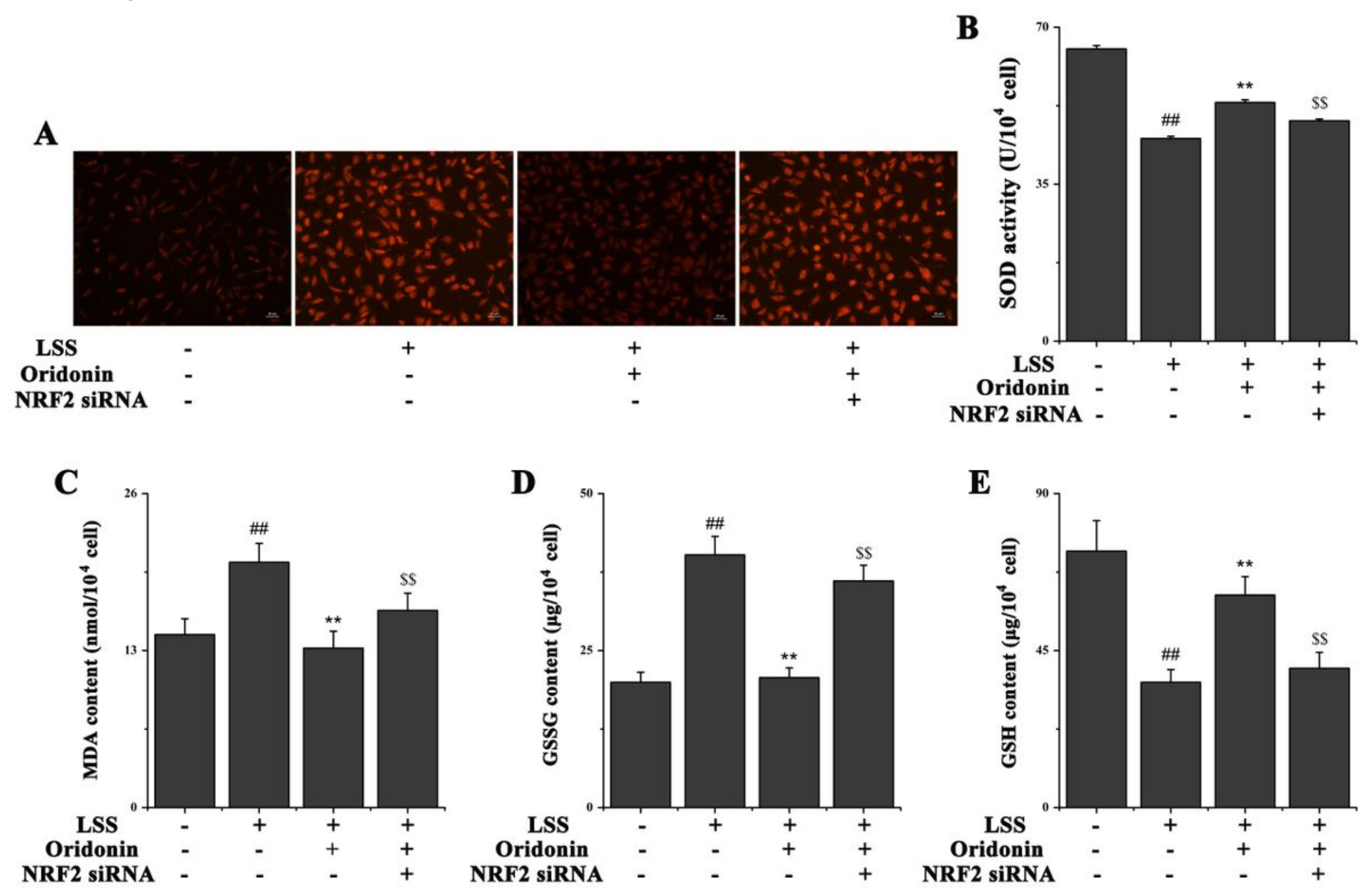

Figure 3

Oridonin alleviates LSS-induced oxidative stress by activating NRF2. (A) Results of the fluorescent probe dye DHE illustrated that oridonin treatment attenuated the LSS-induced increase in ROS activity in EA.hy926 cells. (B) Oridonin treatment increased the LSS-induced decrease in SOD activity in EA.hy926 cells. (C) Oridonin treatment decreased the LSS-induced increase in MDA content in EA.hy926 cells. (D) Oridonin treatment decreased the LSS-induced increase in GSSG content in EA.hy 926 cells. (E) Oridonin treatment increased the LSS-induced decrease in GSH content in EA.hy926 cells. \#\#P<0.01 vs. LSS $(-)+$ oridonin $(-)+$ NRF2 siRNA (-); **P<0.01 vs. LSS (+) + oridonin (-) + NRF2 siRNA (-); \$\$P $<0.01$ vs. LSS (+) + oridonin (+) + NRF2 siRNA (-). LSS, low shear stress; NRF2, nuclear factor erythroid 2-related factor 2; ROS, reactive oxygen species; SOD, superoxide dismutase; MDA, malondialdehyde; GSH, glutathione; GSSG, glutathione disulfide; siRNA, small interfering RNA. 


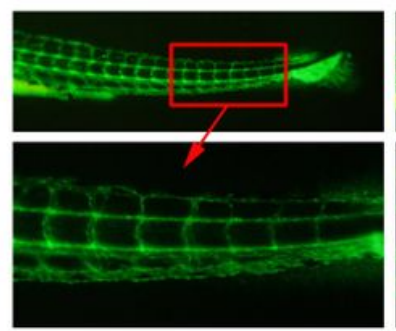

Control

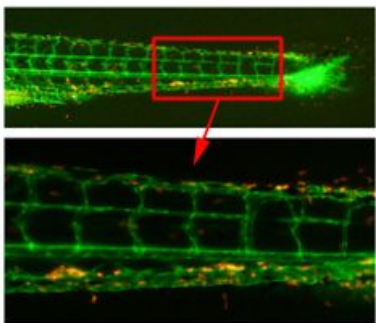

AS
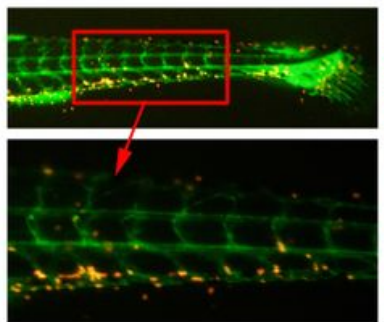

1
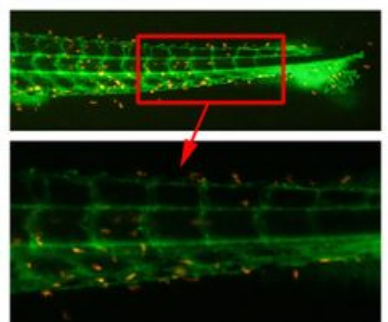

50
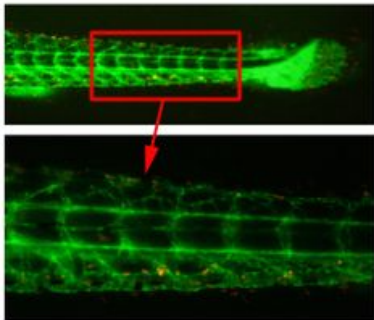

100

Oridonin $(\mu \mathrm{g} / \mathrm{mL})$

Figure 4

Oridonin reduces the formation of early plaques in AS zebrafish blood vessels. AS, atherosclerosis.

$\mathbf{A}$

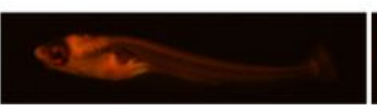

Control

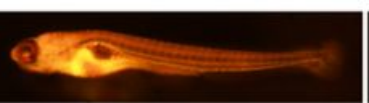

AS

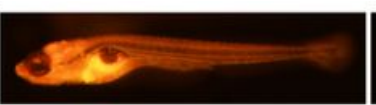

1

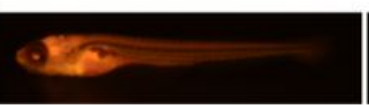

50

Oridonin $(\mu \mathrm{g} / \mathrm{mL})$

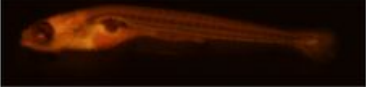

100
B

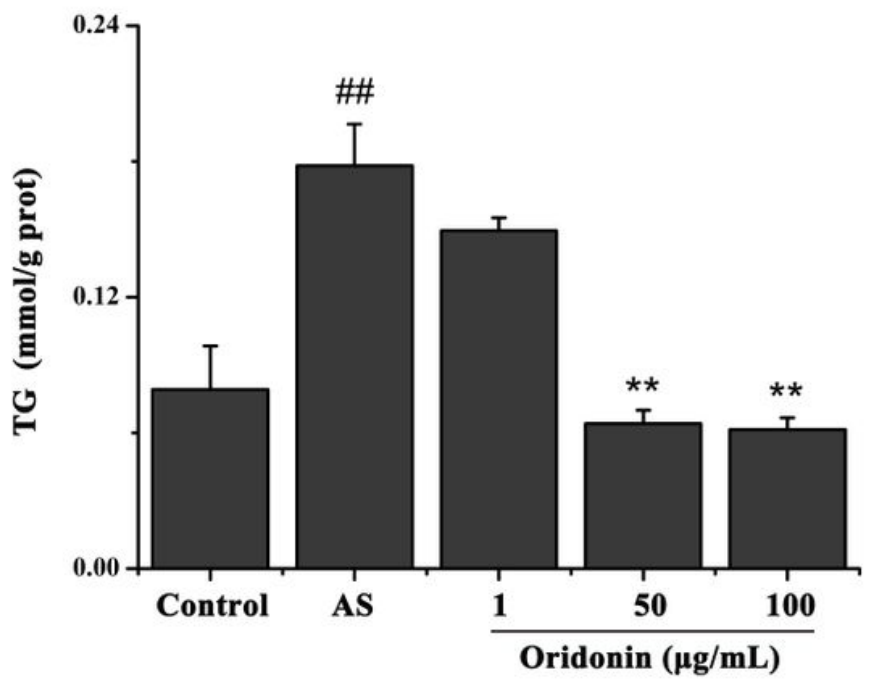

C

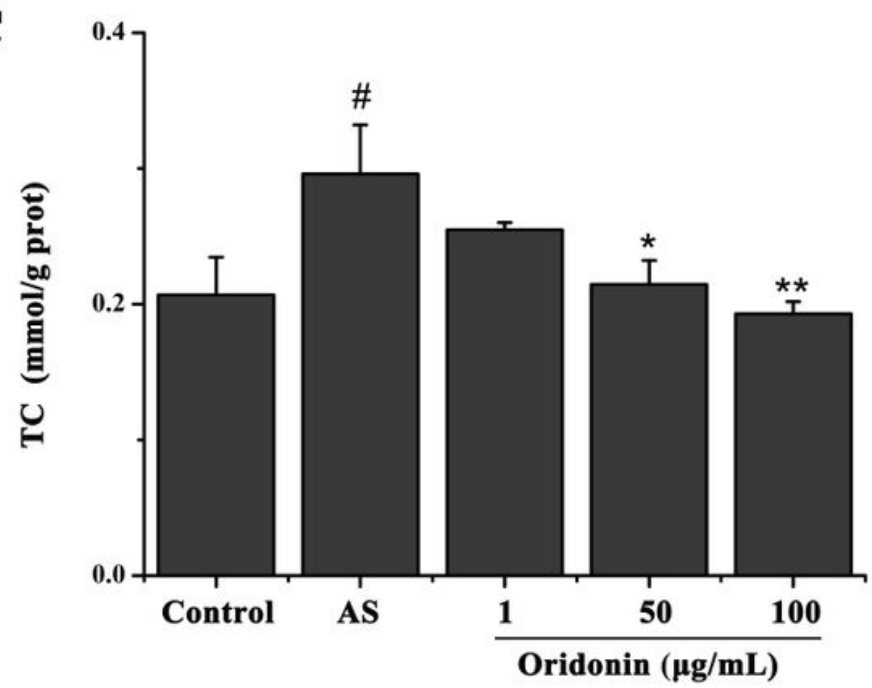

Figure 5

Oridonin reduces the lipid accumulation in a zebrafish AS model. (A) Results of the Nile Red staining revealed that oridonin treatment reduced the blood lipid levels in AS model zebrafish. Oridonin treatment reduced the (B) TG and (C) TC content in AS model zebrafish. \#P<0.05, \#\#P<0.01 vs. control group; ${ }^{\star} \mathrm{P}<0.05,{ }^{*} \mathrm{P}<0.01$ vs. AS group. AS, atherosclerosis; $T G$, triglyceride; TC, total cholesterol. 
$\mathbf{A}$

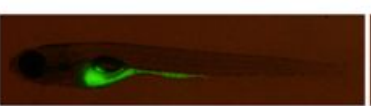

Control

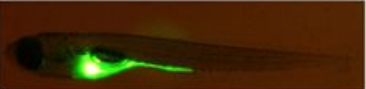

AS

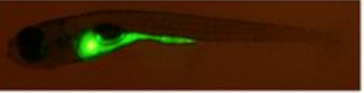

1

C

50

100

Oridonin $(\mu \mathrm{g} / \mathrm{mL})$

B

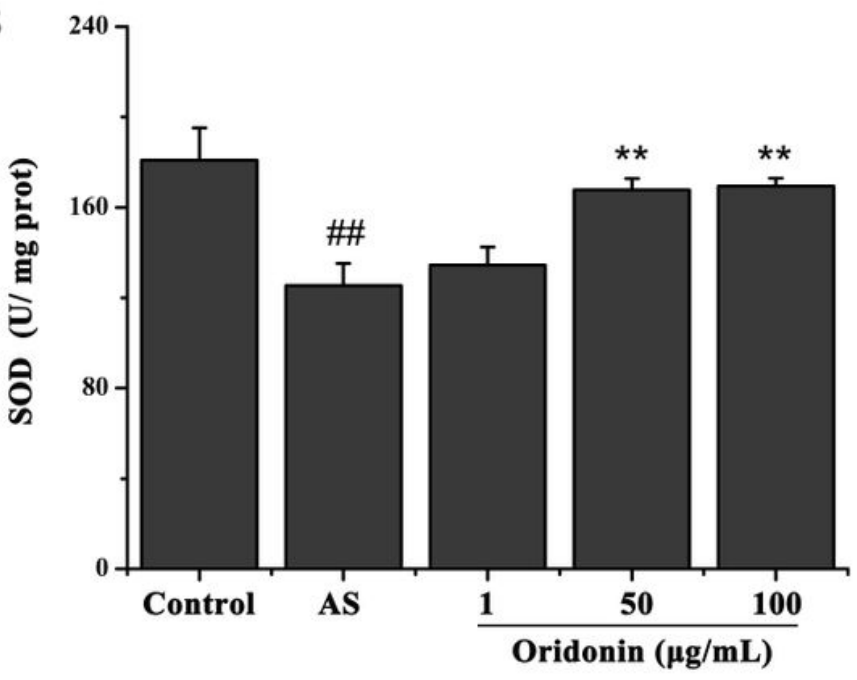

C

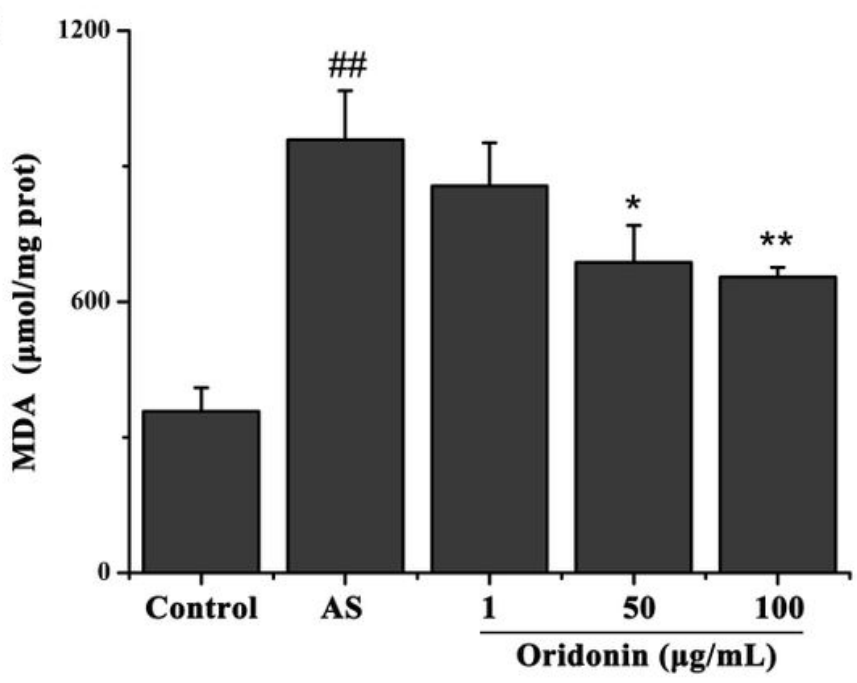

Figure 6

Oridonin reduces the levels of oxidative stress in a zebrafish AS model. (A) Results of the dichlorodihydro-fluorescein diacetate assay revealed that oridonin treatment reduced the ROS activity in the zebrafish AS model. (B) Oridonin treatment increased the SOD activity in the zebrafish AS model. (C) Oridonin treatment reduced the MDA content in the zebrafish AS model. \#\#P<0.01 vs. control group; ${ }^{*} \mathrm{P}<0.05,{ }^{*} \mathrm{P}<0.01$ vs. AS group. AS, atherosclerosis; ROS, reactive oxygen species; $S O D$, superoxide dismutase; MDA, malondialdehyde.

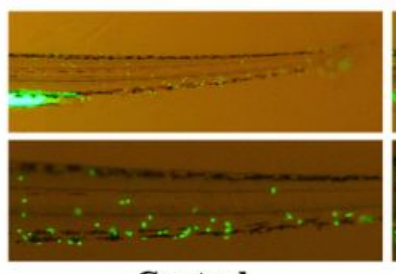

Control

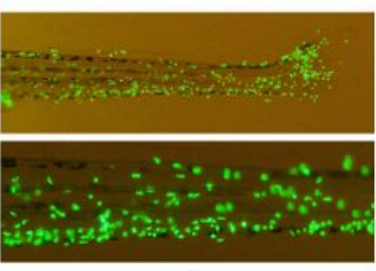

AS

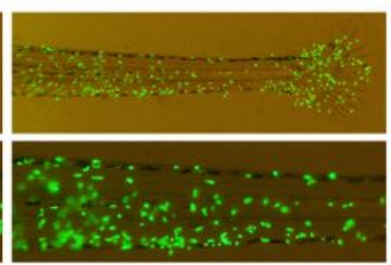

1

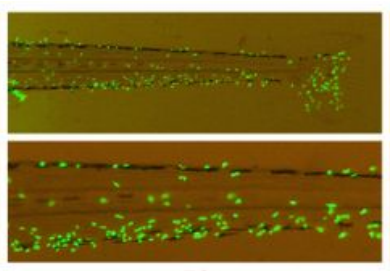

50

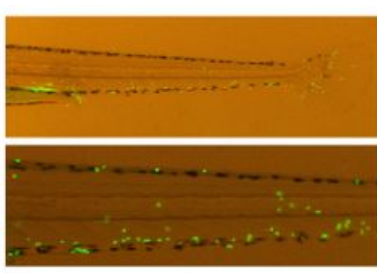

100

Oridonin $(\mu \mathrm{g} / \mathrm{mL})$

\section{Figure 7}

Oridonin reduces the number of neutrophils in a zebrafish atherosclerosis model.

\section{Supplementary Files}

This is a list of supplementary files associated with this preprint. Click to download.

- FigS1.jpg 